\title{
Enhancing Surgical Team Performance with Game-Based Training
}

\author{
Christine Kreutzer ${ }^{1}$, Madeline Marks ${ }^{2}$, Clint Bowers ${ }^{3}$, Curtiss Murphy ${ }^{4}$ \\ 1,2,3 University of Central Florida \\ 1,2 \{christine_kreutzer, marks.madeline\}@knights.ucf.edu \\ 3clint.bowers@ucf.edu \\ ${ }^{4}$ Alion Science and Technology, cmurphy@alionscience.com
}

\begin{abstract}
A striking $70 \%$ of all medical errors are attributed to breakdowns in the interactions amongst medical team members. While many traditional training approaches have sought to mitigate these issues, few have been met with success. Therefore, the purpose of the present study is to explore the use of a serious game to train team communication skills in order to improve patient safety. The results suggest that a game-based approach to training these skills appears to hold promise as a training intervention.
\end{abstract}

Keywords: teamwork, healthcare, serious games, patient safety

\section{Introduction}

According to the Institute of Medicine (IOM), up to 690,000 patients are affected by medical errors each year in the United States, and up to 98,000 of them will die from those errors [1]. There are many root causes for these mishaps, including human error, poor teamwork, and ineffective communication $[2,3]$. In military medicine, these causes are exacerbated by lengthy deployments, which accelerate the decay of both technical and non-technical skills [4]. Studies from both the IOM and the Military Health System (MHS) have concluded that most of these errors can be prevented by adopting patient safety protocols [4]. Patient safety protocols rely on simple techniques such as checklists, team communication, timeouts, sterile protocols, and counting equipment prior to closing [4].

Given the interdisciplinary nature of healthcare, successful team communication is critical to ensuring patent safety. While all team members share the common goal of quality and safety in patient care, they must also perform interdependent tasks while functioning in distinct roles. Team capability typically supersedes individual capability, especially when each team member knows his or her own responsibilities, as well as the responsibilities of all other team members [5, 6]. However, as teamwork is not a result of simply co-locating individuals together, assembling a team does not guarantee effective team operation. Rather, it relies on the members' willingness and ability to communicate, cooperate, and coordinate while remaining focused on the shared goal of patient care [7].

Effective teams must successfully complete both taskwork and teamwork, which is sustained by a commitment to a shared set of team knowledge, skills, and attitudes (KSAs) $[8,9,10]$. Taskwork is related to the performance of specific work-related duties that must be performed by individual members as a function of the organizational role in order to achieve the team goal [11]. On the contrary, teamwork is related to shared behaviors (i.e., what team members do), attitudes (i.e., how team members feel or what they believe), and cognitions (i.e., what team members think or know) required to accomplish the aforementioned tasks [12]. Both taskwork and teamwork are essential to team performance, as they facilitate each other. While taskwork often becomes the key focus of each individual team member, it is teamwork that ensures quality task performance. Despite extensive task knowledge, team goals (i.e. patient care) will not be met unless the team can also successfully communicate, share knowledge, trust one another, and coordinate behaviors [13]. 


\subsection{Barriers to Effective Team Communication}

With the interdisciplinary nature of teamwork comes an imbalance of power. This is especially true in military medicine, where the issue of rank is overlaid upon experience and role. Power, has been defined as the ability of an individual member of an organization to direct or influence the behavior of other members $[14,15]$ thereby impeding the upward flow of information in the organization [16]. Although the notion of teamwork suggests a group working together, the nature of teams in the operating room (OR) present a unique challenge. More specifically, surgeons have a vast level of organizational power relative to fellow team members. These power discrepancies may affect lowpower members' perceptions of the ease of speaking up, thus hindering open discussion. In teams, low-power members accede to those with greater power to avoid rejection or marginalization [17, 18]. In environments in which formal power differences exist and speaking up may influence performance outcomes, those in high-power positions must find ways to minimize silence effects $[19,16]$. Additionally, some team members believe that their input is not needed and sought after by others. Without an explicit reason to provide input, the risks associated with speaking up make it unlikely to occur, even without power discrepancies. Moreover, even if team members are not intimidated by power differences or crippled by the fear of their input being rejected or ridiculed, it is possible that the team member simply does not hone the skills to do so in an appropriate manner, thus preventing them from speaking up [20].

\subsection{Team Training in Healthcare}

Team training provides a learning environment rich in opportunities to practice and receive feedback on newly acquired team skills [21]. Many different approaches to team training have been taken [22].The training design is dependent on many factors, such as the knowledge, skills, and attitudes (KSAs) required for the task, and the available resources. Team training is most robust when available tools, delivery methods, and content are combined [23].

There is a large and growing body of literature supporting the efficacy of teamwork training programs in improving a variety of team competencies and organizational outcomes (e.g., safety and quality within healthcare) [24,22]. Recent work has demonstrated the effect of team training programs on safety and quality metrics. For example, the Veteran's Affairs Medical Team Training program has been shown to decrease preoperative delays, increase antibiotic prophylaxis compliance, decreased equipment issues, decrease handoff issues, and most notably, reduce mortality [25, 26, 27]. These findings are consistent with the broader team training evaluation literature; meta-analytic reviews have demonstrated that team training can account for up to a $20 \%$ improvement in team performance [28, 29].

\subsection{Promoting Teamwork via Game-Based Training}

Serious games are synthetic learning environments (SLEs) which provide a balance of fun, challenge, and learning. According to Michael and Chen [30], "A serious game is a game in which education (in its various forms) is the primary goal, rather than entertainment". Since the launch of the Serious Games Initiative in 2002, there has been a surge in the uptake of games for learning and training across most major industries [31]. A primary rationale for the use of game-based training is that it engages trainees in simulated complex decision-making processes, such as those required in medical training [30]. Another rationale is that the qualities of an effective game are in alignment with the qualities that are required for effective training in general. Specifically, this includes the Laws of Learning, such as recency, exercise, effect, and intensity [32]. Games can do this by leveraging techniques that promote flow such as clear tasks, immediate feedback, no distractions, and a balanced, attainable goal. This is furthered by the use of design techniques promoting intrinsic motivation, simplicity, practice, and limited choice [33]. Another rationale is that games promote additional engagement with the content- the increased time-on-task results in greater learning and self-efficacy [34].

However, as pointed out by Cannon-Bowers and Bowers [35], games among other SLEs often fall short of their intended purpose. In fact, the authors state that “...painfully few attempts have been made to systematically develop a learning environment that is not only well engineered from the technological standpoint, but also built to be consistent with current learning science. Rather, SLEs are almost always built with an emphasis on technology, and pedagogy is often worked in as an afterthought" (p.319). As such, there have been conflicting findings regarding the efficacy of serious games. For example, a review [36] found that 38 out of 67 studies found no significant differences 
in learning outcomes between serious games and traditional training methods. Conversely, a metaanalysis [37] found that games and interactive simulation were more dominant for cognitive gain outcomes as compared to traditional methods. Similarly, a review [38] found that three out of four studies showed that serious games increased cognitive gains as compared to traditional methods. While these mixed findings suggest that caution should be taken when drawing conclusions regarding the efficacy of game-based training, there is substantial evidence and theoretical justification (See Cannon-Bowers and Bowers [35]) to suggest that when carefully and skillfully designed, serious games can have the potential to be effective training tools.

In contrast to traditional training methods, game-based training facilitates experiential learning which requires trainees to gain new knowledge and acquire new skills within a meaningful context in order to build accurate mental models of the complex environment $[39,40]$. Once these mental models have been created, trainees are much more adept to applying their new knowledge and/or skills to novel situations, contexts, or problems [41]. Within the context of teamwork, the role of mental models in coordination, communication, and ultimately team performance is of utmost interest. The members of a well-coordinated team have strong and accurate expectations of what each other will do, and can accurately explain each other's behaviors once they occur [42]. Similarly, communication performance is enhanced if members of the team know what communications are expected of them and can accurately explain communications that they receive [43]. In other words, if team members know what to expect and can explain what they observe, team performance is likely to be enhanced. How this is accomplished is likely to depend on the ways in which tasks are designed, how teams are trained, and how they are prepared for performance in terms of briefings and development of contingency plans. Well-developed and supported mechanisms for forming expectations and explanations are likely to enable streamlined communication, almost implicit coordination and, in the extreme, team members inferring each other's perceived situations [43]. Thus, we suggest that it is reasonable to explore if a game-based training has the potential to improve team communication. In particular, we hypothesize the following:

Hypothesis 1: Those in the game-first condition will perform significantly better those in the knowledge test-first condition on a declarative knowledge test.

Hypothesis 2: Those in the game-first condition will have significantly higher levels of training transfer than those in the knowledge test- first condition.

\section{Methods}

\subsection{Intervention Description}

Safe Surgery Trainer (SST) is a serious game developed by ALION Science and Technology for the United States Navy. As the Navy's first patient safety training game for medical teams involved in surgery- before, during and after, SST provides virtual training scenarios to reinforce safety protocols, enhance team performance and improve outcomes for patients. SST is a single-player game which enables medical personnel to practice virtual training scenarios designed to improve teamwork skills, cross monitoring, and outcomes. SST's immersive environment allows participants to practice patient safety objectives by experiencing each other's roles (i.e., nurse, surgeon, resident) throughout several scenarios. While the scenarios do not increase in difficulty, new content is presented and practiced from the perspective of a different medical team member. This is intended to increase the uptake of patient safety protocols, particularly TeamSTEPPS, which is accepted throughout the military health system. Throughout each module, players are given a dialogue and asked to select the correct response to the virtual team member with whom they are interacting. The players receive feedback regarding each response. First of all, players are presented with two bars at the top of the screen; players receive an increase in the green bar for a correct response, and an increase in the red bar for an incorrect response. Secondly, players receive written feedback. This process feedback provides the player with a brief explanation regarding why the response was correct or incorrect. If the player fails a scenario, they must re-do the module and demonstrate mastery before moving onto the next scenario.

\subsection{Participants}

Participants were non-medical professionals recruited from a large southeastern simulation conference. The final sample included 141 participants. Of these, $25.5 \%$ were female, with an 
average age of 41.36 years $(\mathrm{SD}=13.49)$. Initial analyses revealed that there were no significant differences for gender, age, or hours spent gaming each week between conditions.

\subsection{Design and Procedure}

Individuals were invited in-person to participate in the study. Upon reading and agreeing to the terms outlined in the informed consent, participants were randomized to one of two conditions. One condition involved playing the game first before taking the knowledge test $(n=71)$, and the other group took the knowledge test first and had the option of playing the game afterward $(n=70)$.

\subsection{Measures}

Declarative Knowledge. A declarative knowledge test was constructed following the completion of the SST prototype by creating a bank of questions reflecting the game's content. More specifically, the items were designed to measure knowledge of the teamwork and team communication learning objectives of the SST game. A total of 9 items were constructed. However, 3 items were excluded from the analysis due to a ceiling effect, leaving a total of 6 items (see appendix). Each correct answer earns a total of 1 point. Thus, 6 possible points could be earned by selecting the correct answers in a series of multiple choice items.

Skill Transfer. Although trainees may acquire new knowledge during the training, an increase in basic knowledge is usually not sufficient for the program to be considered successful [44]. Instead, the positive transfer of training, (i.e., the extent to which the learning can be applied to the job and thus results in relevant changes performance) is the primary objective [45, 46]. A total of 7 vignettes representing novel situations in the operating room were created following the completion of the SST prototype to measure training transfer (see appendix). A total of 7 possible points could be earned by selecting the behavior that would promote effective communication in each scenario.

\section{Results}

\subsection{Declarative Knowledge}

An independent-samples t-test was conducted to compare declarative knowledge scores (see figure 1) between the game-first and knowledge test-first conditions. There was a significant difference in the scores between the game-first $(\mathrm{M}=4.25, \mathrm{SD}=1.04)$ and the knowledge test-first $(\mathrm{M}=2.20, \mathrm{SD}=$ 1.01) conditions; $\mathrm{t}(139)=5.61, \mathrm{p}=.000$.

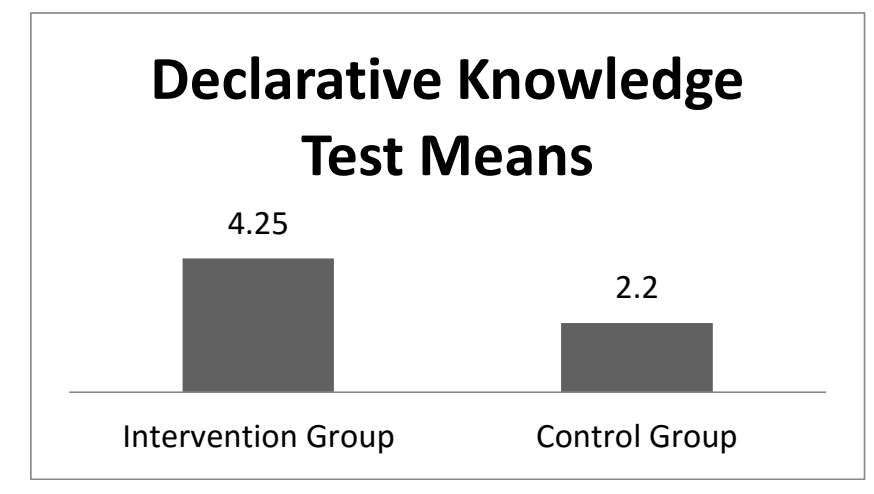

Figure 1. Declarative knowledge test means 
Table 1. Distribution of correct and incorrect responses to declarative knowledge test items.

\begin{tabular}{l|c|c|c|c}
\hline \multicolumn{1}{c|}{$\begin{array}{c}\text { Declarative Knowledge Test } \\
\text { Question }\end{array}$} & $\begin{array}{c}\text { Interventio } \\
\text { n Pre-Test } \\
\text { Percentage } \\
\text { Incorrect }\end{array}$ & $\begin{array}{c}\text { Interventio } \\
\text { n Post-Test } \\
\text { Percentage } \\
\text { Correct }\end{array}$ & $\begin{array}{c}\text { Control } \\
\text { Pre-Test } \\
\text { Percentage } \\
\text { Incorrect }\end{array}$ & $\begin{array}{c}\text { Control Post- } \\
\text { Test } \\
\text { Percentage } \\
\text { Correct }\end{array}$ \\
\hline "What is one way of speaking up?" & $7.4 \%$ & $92.6 \%$ & $25.0 \%$ & $75.0 \%$ \\
\hline $\begin{array}{l}\text { "Ellen is agitated that you keep } \\
\text { asking questions during the tasking } \\
\text { meeting. You would explain to her } \\
\text { that questions," }\end{array}$ & $16.2 \%$ & $83.8 \%$ & $29.8 \%$ & $70.2 \%$ \\
\hline "An example of a safety word is..." & $12.8 \%$ & $87.2 \%$ & $28.2 \%$ & $71.8 \%$ \\
\hline $\begin{array}{l}\text { "In the acronym CUS, what do the } \\
\text { U and the S stand for?" }\end{array}$ & $25.5 \%$ & $74.5 \%$ & $89.9 \%$ & $10.1 \%$ \\
\hline $\begin{array}{l}\text { "Which is an example of an open } \\
\text { ended question?" }\end{array}$ & $25.8 \%$ & $74.2 \%$ & $61.1 \%$ & $38.9 \%$ \\
\hline $\begin{array}{l}\text { "Why is it important to use open- } \\
\text { ended questions versus yes/no } \\
\text { questions?" }\end{array}$ & $12.4 \%$ & $87.6 \%$ & $20.0 \%$ & $80.0 \%$ \\
\hline
\end{tabular}

\subsection{Training Transfer}

An independent-samples t-test was conducted to compare level of training transfer (see figure 2) between the game-first and knowledge test- first conditions. There was a significant difference in the scores for the game-first $(M=6.94, S D=1.76)$ and the knowledge test-first $(M=5.79, S D=1.66)$ conditions; $\mathrm{t}(139)=4.13, \mathrm{p}=.000$.

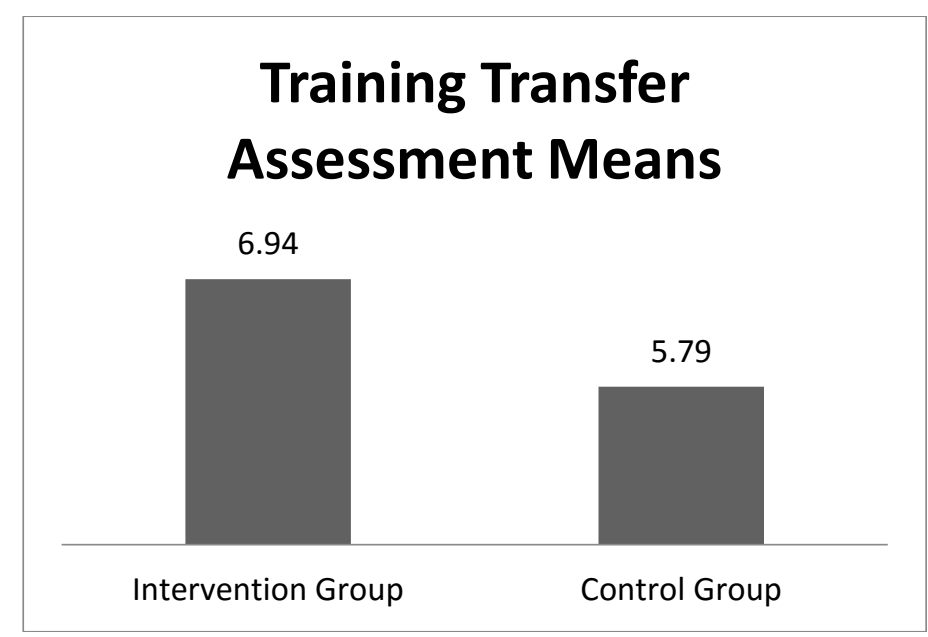

Figure 2. Training transfer assessment test means 
Table 2. Distribution of correct and incorrect responses to skill transfer items.

\begin{tabular}{|c|c|c|c|c|}
\hline Skill Transfer Question & $\begin{array}{l}\text { Intervention } \\
\text { Pre-Test } \\
\text { Percentage } \\
\text { Incorrect }\end{array}$ & $\begin{array}{c}\text { Interventio } \\
\text { n Post-Test } \\
\text { Percentage } \\
\text { Correct }\end{array}$ & $\begin{array}{l}\text { Control } \\
\text { Pre-Test } \\
\text { Percentage } \\
\text { Incorrect }\end{array}$ & $\begin{array}{l}\text { Control } \\
\text { Post-Test } \\
\text { Percentage } \\
\text { Correct }\end{array}$ \\
\hline $\begin{array}{l}\text { "You are a member of the HR team and } \\
\text { you are the last person to meet with the } \\
\text { new hire, Jose, to complete the paper } \\
\text { work process. You ask Jose for his } \\
\text { employee ID number. Jose becomes } \\
\text { visibly annoyed and asks, "Why do I have } \\
\text { to keep giving you my employee ID } \\
\text { number, it is right there on top of my } \\
\text { file!" You explain to Jose that good } \\
\text { communication," }\end{array}$ & $12.9 \%$ & $87.1 \%$ & $27.9 \%$ & $72.1 \%$ \\
\hline $\begin{array}{l}\text { "At work, your colleague Jennifer notices } \\
\text { that George did not properly lock the } \\
\text { door. Jennifer tells her colleague, "I think } \\
\text { that there might be something wrong with } \\
\text { how George locked the door." Jennifer's } \\
\text { colleague brushes off the statement and } \\
\text { continues working. What would you } \\
\text { recommend that Jennifer say to } \\
\text { strengthen her reporting of the problem?" }\end{array}$ & $13.0 \%$ & $87.0 \%$ & $31.9 \%$ & $68.1 \%$ \\
\hline $\begin{array}{l}\text { "Now that Jennifer has properly informed } \\
\text { her colleague that George did not lock the } \\
\text { door properly what should happen next?" }\end{array}$ & $4.5 \%$ & $95.5 \%$ & $15.3 \%$ & $84.7 \%$ \\
\hline $\begin{array}{l}\text { "There is a new project manager, Bart. } \\
\text { The previous project manager was fired } \\
\text { because the team did not know what role } \\
\text { each person was supposed to play. In } \\
\text { order to create a more successful } \\
\text { environment..." }\end{array}$ & $51.2 \%$ & $48.8 \%$ & $54.3 \%$ & $45.7 \%$ \\
\hline $\begin{array}{l}\text { "Bart, the project manager, has asked } \\
\text { everyone to go around and introduce } \\
\text { themselves. It is your turn to introduce } \\
\text { yourself, what should you say?" }\end{array}$ & $12.5 \%$ & $87.5 \%$ & $23.6 \%$ & $82.5 \%$ \\
\hline $\begin{array}{l}\text { "Someone comes up to you while you are } \\
\text { in the middle of an important task and } \\
\text { asks you for help with a less important } \\
\text { task. You tell them, "I'm just finishing } \\
\text { this up. I can help you in } 10 \\
\text { minutes." This is an example of what } \\
\text { style of communication?" }\end{array}$ & $85.6 \%$ & $14.4 \%$ & $80.8 \%$ & $19.2 \%$ \\
\hline
\end{tabular}




\begin{tabular}{l|l|l|l|l}
\hline $\begin{array}{l}\text { "You approach a colleague about an error } \\
\text { she made. She accuses you of picking on } \\
\text { her. An assertive response would be," }\end{array}$ & $4.4 \%$ & $95.6 \%$ & $6.8 \%$ & $93.2 \%$ \\
\hline
\end{tabular}

\section{Discussion}

Taken as a whole, these results converge to support the hypotheses that a game-based training approach can be effective in teaching knowledge of and improving transfer of behaviors that facilitate effective team communication. More specifically, it appears as though the form of practice and feedback provided by SST has the potential to facilitate team skills that could improve patient safety in the operating room. Participants who played the game demonstrated higher levels of declarative knowledge about effective communication behaviors. Those who played the game were also better able to apply knowledge about effective team communication to novel situations, and displayed higher levels of training transfer in comparison to those who took the knowledge test first. Thus, playing the game was indeed helpful. This suggests that in addition to increased knowledge, behavioral changes are possible if a game delivers appropriate information and provides opportunities for practice. Moreover, the feedback provided by SST allows players to correct their responses and practice target behaviors appropriately in order to facilitate positive transfer.

Although the results largely supported the hypotheses, there are shortcomings to the present design that require the data to be interpreted with caution. Although it would be ideal to generalize to surgical teams, the present data were collected on a sample of conference attendees with a penchant for simulation, which may limit the applicability of the results. It should be noted that game-based training has been shown to be an effective approach in training other knowledge and skills [47]. Thus, paired with the foundation laid by this study, it would be reasonable to replicate this study in the target population in order to determine whether these results generalize. It should also be noted that the present study used a control group that had the option to play the game after completing the instructional effectiveness measures. Thus, conclusions are unable to be drawn about the effectiveness of the game compared with traditional forms of training. Using other comparison groups, such as including a condition that received a lecture-based training or a condition that featured a format for practice such as live exercises, might provide greater insight into the use and effectiveness of this game. With that said, these data suggest that a serious game can be an effective way to provide training to improve team communication skills. It is the researchers' hope that this report sparks greater interest in this approach.

\section{References}

[1] Schulz, K. F., Altman, D. G., \& Moher, D. (2010). CONSORT 2010 statement: updated guidelines for reporting parallel group randomised trials. BMC medicine, 8(1), 18. http://dx.doi.org/10.1186/1741-7015-8-18

[2] Manser, T. (2009). Teamwork and patient safety in dynamic domains of healthcare: a review of the literature. Acta Anaesthesiologica Scandinavica, 53 (2), 143-151. http://dx.doi.org/10.1111/j.1399-6576.2008.01717.x

[3] Mazzocco, K., Petitti, D. B., Fong, K. T., Bonacum, D., Brookey, J., Graham, S., \& Thomas, E. J. (2009). Surgical team behaviors and patient outcomes. The American Journal of Surgery, 197(5), 678-685. http://dx.doi.org/10.1016/j.amjsurg.2008.03.002

[4] US DoD. (2008). TeamSTEPPS Goes To War. Patient Safety Program Newsletter, (2008).

[5] Smith-Jentsch, K. A., Salas, E., \& Baker, D. P. (1996). Training team performance-related assertiveness. Personnel Psychology, 49(4), 909-936. http://dx.doi.org/10.1111/j.17446570.1996.tb02454.x

[6] Volpe, C. E., Cannon-Bowers, J. A., Salas, E., \& Spector, P. E. (1996). The impact of crosstraining on team functioning: An empirical investigation. Human Factors: The Journal of the Human Factors and Ergonomics Society, 38(1), 87-100. http://dx.doi.org/10.1518/001872096778940741

[7] Salas, E., \& Frush, K. (2012). Improving patient safety through teamwork and team training. Oxford University Press. 
[8] Morey, J. C., Simon, R., Jay, G. D., Wears, R. L., Salisbury, M., Dukes, K. A., \& Berns, S. D. (2002). Error reduction and performance improvement in the emergency department through formal teamwork training: evaluation results of the MedTeams project. Health services research, 37(6), 1553-1581. http://dx.doi.org/10.1111/1475-6773.01104

[9] Burke, C. S., Wilson, K. A., \& Salas, E. (2003). Teamwork at 35,000 feet: Enhancing safety through team training. Human Factors and Aerospace Safety.

[10] Salas, E., Shuffer, M. L., Thayer, A. L., Bedwell, W. L., \& Lazzara, E. H. (2014). Understanding and diagnosing teamwork in organizations: a scientifically-based practical guide. Hum Resour Manage.

[11] Wildman, J. L., Thayer, A. L., Rosen, M. A., Salas, E., Mathieu, J. E., \& Rayne, S. R. (2012b). Task types and team-level attributes: Synthesis of team classification literature. Human Resource Development Review, 11, 97-129. http://dx.doi.org/10.1177/1534484311417561

[12] Morgan, B.B., Jr., Salas, E., \& Glickman, A.S. (1994). An analysis of team evolution and maturation. The Journal of General Psychology, 120, 277-291. http://dx.doi.org/10.1080/00221309.1993.9711148

[13] Mathieu, J., Maynard, M. T., Rapp, T., \& Gilson, L. (2008). Team effectiveness 1997-2007: A review of recent advancements and a glimpse into the future. Journal of Management, 34(3), 410-476. http://dx.doi.org/10.1177/0149206308316061

[14] Kanter, R. M. (1979). Differential access to opportunity and power. Discrimination in organizations, 52, 69.

[15] Pfeffer, J. (1981). Power in organizations (Vol. 33). Marshfield, MA: Pitman.

[16] Wu, F., Huberman, B. A., Adamic, L. A., \& Tyler, J. R. (2004). Information flow in social groups.Physica A: Statistical Mechanics and its Applications, 337(1), 327-335. http://dx.doi.org/10.1016/j.physa.2004.01.030

[17] Estrada, M., Brown, J. and Lee, F. (1995). 'Who gets the credit? Perceptions of idiosyncrasy credits in work groups'. Small Groups Research, 26, 1, 56-76. http://dx.doi.org/10.1177/1046496495261003

[18] Hoffman, L. R., \& Maier, N. R. (1961). Quality and acceptance of problem solutions by members of homogeneous and heterogeneous groups. The Journal of Abnormal and Social Psychology, 62(2), 401. http://dx.doi.org/10.1037/h0044025

[19] Dépret, E., \& Fiske, S. T. (1993). Social cognition and power: Some cognitive consequences of social structure as a source of control deprivation. In Control motivation and social cognition (pp. 176-202). Springer New York. http://dx.doi.org/10.1007/978-1-4613-8309-3_7 
* Indicates transfer item

*1: You are a member of the HR team and you are the last person to meet with the new hire, Jose, to complete the paper work process. You ask Jose for his employee ID number. Jose becomes visibly annoyed and asks, "Why do I have to keep giving you my employee ID number, it is right there on top of my file!" You explain to Jose that good communication, a. "minimizes mistakes."

b. "is part of my job."

c. "is written in the protocol."

d. "often can be annoying."

2: What is one way of speaking up?

a. Talking loudly

b. Writing a note

c. Speaking slowly

d. Asking questions

3: Ellen is agitated that you keep asking questions during the tasking meeting. You would explain to her that questions,

a. "demonstrate that you are listening."

b. "help uncover hidden problems."

c. "demonstrate that you care."

d. "help you understand the problem better."

4: An example of a Safety word is...

a. Worried

b. Concerned

c. Troubled

d. Careless

5: In the acronym CUS, what do the $U$ and the $S$ stand for?

a. Urgent and Safety

b. Uncomfortable and Sterility

c. Uncomfortable and Safety

d. Urgent and Sterility

6: Which is an example of an open ended question?

a. Do you think you should wait?

b. What might happen if you wait?

c. When will you speak to her?

*7: At work, your colleague Jennifer notices that George did not properly lock the door. Jennifer tells her colleague, "I think that there might be something wrong with how George locked the door." Jennifer's colleague brushes off the statement and continues working. What would you recommend that Jennifer say to strengthen her reporting of the problem?

a. "I am worried that George did not lock the door properly and it makes me uneasy and this jeopardizes our security."

b. "I am troubled that George did not lock the door properly and it makes me uneasy and this jeopardizes our sterility."

c. "I am concerned that George did not lock the door properly and it makes me uncomfortable and this jeopardizes our safety."

d. "I think that George is careless and did not lock the door properly and it makes me nervous and this jeopardizes our sanity."

*8: Now that Jennifer has properly informed her colleague that George did not lock the door properly what should happen next?

a. George should be reported to his supervisors for not properly locking the door. 
b. The entire work team needs to address the problem of making sure everyone knows how to properly lock the door.

c. The entire work team should be written up for not making sure that George locked the door properly.

d. Jennifer should not be considered trustworthy and an asset to the work team because she called attention to George's mistake.

*9: There is a new project manager, Bart. The previous project manager was fired because the team did not know what role each person was supposed to play. You and your coworkers have not worked with Bart and are concerned that the project might fail for the reasons that the previous project manager was fired. In order to create a more successful environment...

a. You would recommend that the team meet before the project begins to discuss expectations of each team member.

b. You would recommend the creation of an employee manual before each project begins.

c. You would recommend that the team should just give up now because the project is doomed to fail

d. You would recommend that Bart call a team meeting before the project begins to discuss the failings of the previous project manager.

*10: Bart, the project manager, has asked everyone to go around and introduce themselves. It is your turn to introduce yourself, what should you say?
a. Hi, I'm Wanda.
b. Hi, I'm Wanda, the floor manager.
c. Hi, I'm the floor manager

11: Why is it important to use open-ended questions versus yes/no questions?

a. Allows people to talk more

b. Encourages team involvement

c. Lets people feel important and that they are being heard

d. Is a more assertive way of communicating?

*12: Someone comes up to you while you are in the middle of an important task and asks you for help with a less important task. You tell them, "I'm just finishing this up. I can help you in 10 minutes." This is an example of what style of communication

a. Passive

b. Aggressive

c. Assertive

*13: You approach a colleague about an error she made. As you begin to talk to her, she accuses you of picking on her. An assertive response would be,

a. "Let's talk this over and straighten this out."

b. "Don't interrupt me while I'm talking to you."

c. "I'm sorry, we can do this another time." 